\title{
A deadlock-free subnetting mechanism for high performance broadcasting in NoC
}

\author{
Xing Han ${ }^{a)}$ Yuzhuo Fu, Jiang Jiang, and Chang Wang \\ School of Microelectronics, Shanghai Jiao Tong University, \\ Dongchuan Rd. 800, Shanghai, China \\ a)hanxing@sjtu.edu.cn
}

\begin{abstract}
In this letter we present a mechanism to generate subnets for the tasks running on network-on-chip, gaining better performance in broadcast. The present results show that subnetting supplies apparent improvement in the performance of on-chip communications mixed with both unicast and broadcast on manycore processors. However, due to unpredictable task submitting sequence, the applications coming following will be mapped into subnets with different irregular topologies, in which the performance of communications will be restricted by topologies apparently. We propose a flexible subnetting mechanism to build subnet for specified cores on manycore processor to address this problem. Improved hardware support also requires fewer circuits in router design. In this letter, both the unicast and the broadcast traffics are considered. Experiments show that about $10 \%$ improvement in performance over the traditional subnetting method is gained on average.
\end{abstract}

Keywords: broadcast, manycore, subnet, routing, network-on-chip

Classification: Electron devices, circuits, and systems

\section{References}

[1] S. Bell, B. Edwards and J. Amann: ISSCC Dig. Tech. Papers (2008) 88. DOI:10.1109/ISSCC.2008.4523070

[2] K. Kandalla, A. Venkatesh and K. Hamidouche: HOTI (2013) 63. DOI:10. 1109/HOTI.2013.26

[3] B. Dinechin, P. Massas and G. Lager: ICCS (2013) 1654.

[4] N. Jerger, L. Peh and M. Lipasti: ISCA (2008) 229. DOI:10.1109/ISCA.2008. 12

[5] X. Wang, M. Yangm, Y. Jiang and P. Liu: J. MICPRO 35 (2011) 119. DOI:10. 1016/j.micpro.2010.08.003

[6] S. Borkar: DAC (2007) 746. DOI:10.1145/1278480.1278667

[7] D. Kim, K. Daejeon, H. Kim and J. Huh: MICRO (2010) 459. DOI:10.1109/ MICRO.2010.16

[8] W. Dally and C. Seitz: IEEE Trans. Comput. C-36 (1987) 547. DOI:10.1109/ TC.1987.1676939

[9] S. Kumar, A. Jantsch, J. Soininen, M. Forsell, M. Millberg, J. Oberg, K. Tiensyrja and A. Hemani: VLSI (2002) 105. DOI:10.1109/ISVLSI.2002. 
1016885

[10] N. Binkert, B. Beckmann, G. Black, S. Reinhardt and A. Saidi: SIGARCH (2011) 1 .

\section{Introduction}

Nowadays, manycore processor becomes one of the most important trends after the era of multicore processors, such as Tile64 [1], MIC [2] and MPPA-256 [3]. Integrating large quantities of cores brings challenges to the performance of communications in NoC, especially to the performance of broadcasts. Cache coherence in multicore and manycore processors, such as Directory and Token, generates 5-5.5\% multicast and broadcast communications [4]. Wang [5] claims that about $5-10 \%$ on-chip traffics are one-to-many. Reference [4] also shows the NoC saturates with injection rate at $5 \%$ and one-to-many traffics at $10 \%$.

Subnetting is one predictable trend for manycore processors to address this problem. It is also sensible to run different applications on one manycore processor with a large quantities of cores simultaneously, because most applications gain limited benefits using more than 16 cores [6]. Wang [5] proposed an efficient multicasting scheme in manycore processor, dividing the manycore into regular subnets. Kim claims [7] that supporting subnet can improve the performance of cache coherence in manycore processors.

Although these alogrithms bring obvious improvement in performance with initial regular topology, they cannot handle the on-line scheduling. After the scheduling of large mount of applications, cores occupied by one application may get separated over the whole processor.

In this letter, we report a subnet generating algorithm for irregular topologies, gaining around $10 \%$ improvement in performance on average, while speedup is up to $24 \%$ in the best cases compared with traditional subnetting algorithm.

\section{Algorithm design}

In this letter, we report our methodology to determine a better fitted subnet and our deadlock-free routing algorithm.

\subsection{Determining the fitted subnet}

The traditional subnet generating algorithms seek to find the most fitted subnet (MFS) over the cores in one application [5, 7].

However, the number of available links in MFS also decreases. Given a manycore processor with $n$ applications, $N$ cores and each application $P_{i}$ occupies $C_{i}$ cores, and each subnet has $W_{i}$ links. Assume that the ratio of broadcast is $b$ and application $P_{i}$ generates network messages of $M_{i}$, then traffics $T_{i}$ generated in subnet are

$$
T_{i}=b \times\left(C_{i}-1\right) \times M_{i}+(1-b) \times M_{i}=\left(1+b \times C_{i}-2 b\right) \times M_{i} .
$$

Assuming $W_{i} \approx 4 \times C_{i}$ for each subnet and the average link load $L_{i}=T_{i} / W_{i}$, we have 


$$
L_{i}=\frac{\left(1+b \times C_{i}-2 b\right) \times M_{i}}{4 \times C_{i}}=\frac{M_{i}}{4} \times\left(b+\frac{1-2 b}{C_{i}}\right) .
$$

With lower broadcast ratio, enlarging subnet properly will give benefits to the performance.

First we give some definitions for the sake of convenient description. Logical Subnet $(L S)$ is the set of the cores occupied by one application actually. Physical Subnet $(P S)$ is a subnet that is used to provide routing pathes for $L S$. The $P S$ contains two parts: one kernel part $(K P)$ and up to four attached parts $(A P \mathrm{~s}) . K P$ contains most of cores while $A P$ s are mainly used to supply available routing paths. According to these definitions, we have $L S \subseteq P S$ and $P S=K P \cup\left(\cup A P_{i}\right)$.
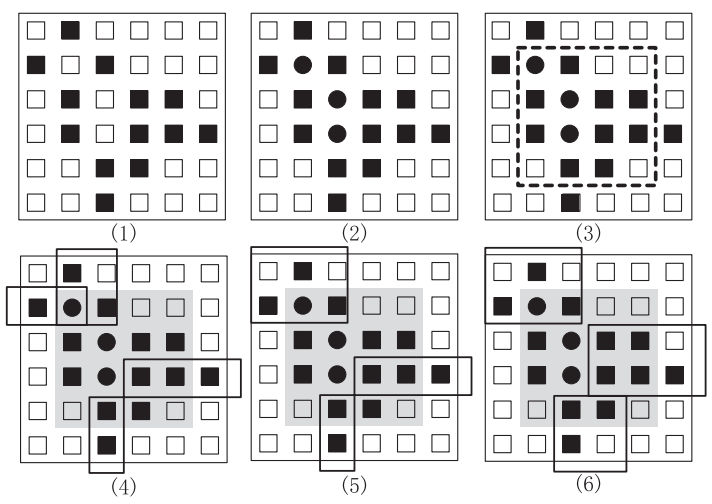

Fig. 1. Determining the fitted subnet

For one $L S$, we determine the $P S(K P$ and $A P \mathrm{~s})$ in the following steps.

1. Let $P S=L S$ as the initial cores set, as is shown in Fig. 1(1).

2. Ensure any two cores in $P S$ have the shortest path in $P S$.

For each row, we can figure out the range of the cores on $\mathrm{X}$-axis, denoting as $\left[X_{\min }, X_{\max }\right]$. Then we add all the cores with $X_{\min }<x<X_{\max }$ to our $P S$ set. The same operation is dealt on each columns. After this step, $P S$ should be like the one in Fig. 1(2).

3. Find the kernel part $(K P)$.

There is a tradeoff in finding the $K P$. As is analyzed in the former section, large $K P$ brings more external cores and links, which means the broadcasts will influence much more area and restrict the performance badly. While $K P$ should cover as many cores in $P S$ as possible to supply more routing pathes to the final $P S$, according to Eq. (2). We will focus on addressing this tradeoff in this step.

1) $X_{\min }=\min (\{x \mid(x, y) \in P S\}), X_{\max }=\max (\{x \mid(x, y) \in P S\})$

2) Denote the range of cores on column $X_{\min }$ as $\left[Y_{X_{\min }, \min }, Y_{X_{\min }, \max }\right]$. Denote the range on column $X_{\max }$ as $\left[Y_{X_{\max }, \min }, Y_{X_{\max }, \max }\right]$

3) Let $Y_{\min }=\max \left(Y_{X_{\min }, \min }, Y_{X_{\max }, \min }\right)$ and $Y_{\max }=\min \left(Y_{X_{\min }, \max }, Y_{X_{\max }, \max }\right)$.

4) Compute the coverage-ratio $(C R)$ and available-cores-ratio $(A R)$ of rectangle $R=X_{\min }, X_{\max }, Y_{\min }, Y_{\max } . C R=\operatorname{num}(R \cap P S) / \operatorname{num}(P S)$ and $A R=$ $\operatorname{num}(R \cap P S) / \operatorname{num}(R)$.

5) Increasing $X_{\min }$ or decreasing $X_{\max }$ by turns, repeat the steps from step 2) 
to step 4) and find the highest $A R$ and the highest $C R$.

6) Computing $L_{i}$ for the rectangles with highest $A R$ and $C R$ according to Eq. (2). Choose the one with lower $L_{i}$ as $K P$. By now, we get the $K P$ shown in Fig. 1(3).

4. Generating attached parts $(A P \mathrm{~s})$.

1) The rest cores on the edge of $K P$ form four rectangles naturally, as is shown in Fig. 1(4). Denote them as $A P_{L}, A P_{R}, A P_{U}$ and $A P_{D}$ in four directions.

2) Combine $A P_{\mathrm{S}}$ sharing overlap. For example, we combine the $A P_{L}$ and $A P_{U}$ into $A P_{L U}$ in Fig. 1(5). This helps to reducing the complexity of routing algorithm.

3) Enlarge the region of $A P \mathrm{~S}$ to obtain lower $L_{i}$ in Eq. (2). In Fig. 1(5), expanding $A P_{R}$ on axis-Y is accepted because of the decrement of $L_{i}$. While expanding $A P_{L U}$ is declined due to the increment of $L_{i}$.

5. The final $P S=K P \cup\left(\cup A P_{i}\right)$.

\subsection{Deadlock-free routing algorithm}

The deadlock-free routing is available for our subnetting.

Dally [8] described a directive principle in deadlock-free routing algorithm design. An $X Y X$ or $Y X Y$ routing may occur in irregular subnet. Additional dependency $C_{Y} \rightarrow C_{X}$ brings the possibility of deadlock in such routing algorithm. we modify $X Y$ routing to fit our algorithm. Rerouting the flit on link on VC $k$ to VC $k+1$ at each $Y X$ routing could efficiently break the potential circled dependency.

\section{Hardware support}

Wang's work describes an efficient hardware support mechanism for any subnet in which any two cores have a shortest path [7]. The original mechanism is adding four bits in every router of NoC to judge whether the current router is on the border of the subnet. When a message comes, it calculates the direction for next hop using MUXes. If the flag of this direction is one, the message will be routed into this direction.
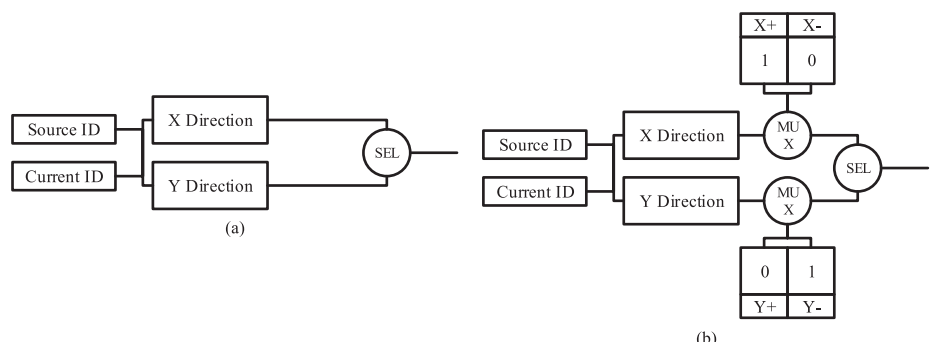

(b)

Fig. 2. Hardware support for subnetting

We make some modifications to make it much simpler. Compared with the $X Y$ Routing Algorithm [9] in Fig. 2(a), only two sets of MUX are added into the circuits on the routine of computing $X$ and $Y$. In the original $X Y$ Routing Algorithm as is shown in Fig. 2(a), the judgment on $Y$ direction is computed after the judgment 
on $X$ direction. While in this letter, results on $X$ direction will be selected by flags $X+$ and $X-$ and results on $Y$ by flags $Y+$ and $Y-$. The result on $X$ direction still has higher priority. With this mechanism, we limit both unicast and broadcast into one subnet, which reduces the influence to the communications of other subnets.

Using VC to avoid deadlock [8], the number of VCs determined the maximum acceptable routing from $Y$ direction back to $X$ direction. With our subnetting mechanism, no more than three $Y X$ routings occur in the complete routing for any flit in our subnetting mechanism, which means deadlock-free could be easily ensured.

\section{Simulation and results}

In our simulation, we run a $8 \times 8$ mesh with fixed pipeline in Gem5 [10]. We compare our algorithm (noted as NEW in results) with traditional most fitted subnet (MFS). Network latency and average load on links are chosen as metrics to evaluate our subnet-generating algorithm.

We use a simple scheduling algorithm, searching the whole $\mathrm{NoC}$ in a random way and find the most nearest cores and return to the subnet-generating algorithm. To get a fair mapping result, we run a long period scheduling and dump the mapping results every 200 times of scheduling. The input data are formed with unicast and broadcast.

To compare with the other researches $[4,5]$, we set the broadcast ratio to be from $5 \%$ to $15 \%$ [4], which covers the situations for cache protocols on manycore processor. The messages injected into NoC use the model built in Gem5 and the injection rate is from 0.1 to 1.0 .

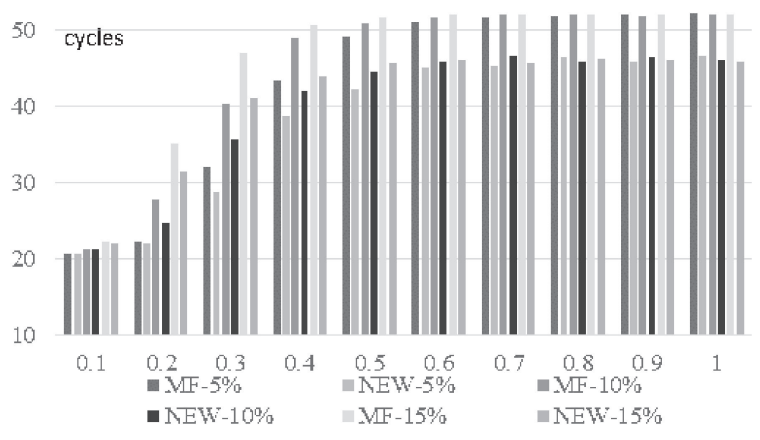

Fig. 3. Average network latency

As is shown in Fig. 3, when injection rate is lower than 0.2, only $0.18-0.91 \%$ improvement in performance is gained. That is because NoC saturates with lower traffics. For injection rate from 0.4 to 0.7 , our algorithm gains the highest benefits around $11-14 \%$. Because NoC saturates far before the injection rate reaches 1.0 with broadcast. When injection rate is higher than $0.7, \mathrm{NoC}$ is over saturated and network latency reaches maximum up to 46 cycles with our algorithm and 52 cycles with the most fitted algorithm. The speedup also drops to around $10 \%$.

As is shown in Fig. 4, the average load on links also shows the same tendency, and the highest benefits are gained with the same injection rate. When the injection 


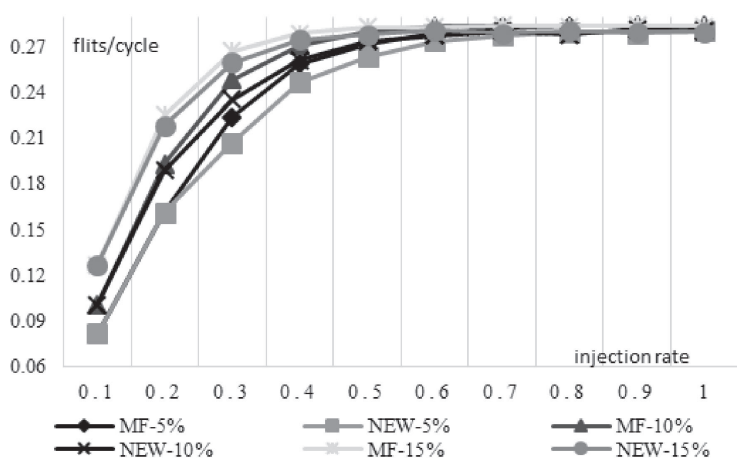

Fig. 4. Average throughput on links

rate is lower than 0.2 or higher than 0.7 , little benefit is gained. When the injection rate is between 0.2 and 0.6 , the speedup is more than $10 \%$.

The best benefits in network latency could be gained in a nearly saturated NoC. Form Fig. 4, we could find that the NoC saturates with injection rate at 0.6 with 5\% broadcasts and at 0.5 with $10 \%$ broadcasts. In Fig. 3, we should notice that the best benefits are gained with injection rate at 0.2 to 0.7 . This means when the NoC is nearly saturated, the benefit still stays in a high level.

Another fact we should find is that benefit is acceptable with $5 \%-10 \%$ broadcasts in nearly saturated NoC. It just meets the range of broadcasts in NoC running cache coherence protocols [5].

\section{Conclusion}

Traditional subnetting algorithms use the most fitted subnet to cover the task group and reduce the amount of available routing paths, which limits the performance of communication in one subnet. This letter suggests a subnet generating algorithm which gains around $10 \%$ improvement in performance on average with $5-15 \%$ broadcasts, which is also the ratio in realistic NoC running cache coherence on it. The best improvements are gained when NoC is near saturated according to our analyses. Two bits of storage for each subnet in router are required in our work, while four are demanded in a previous one.

\section{Acknowledgments}

This work is supported by the National Science Foundation of China (Grant No. 61373032). 